\title{
Influência de diferentes técnicas de extração sobre a capacidade antioxidante do da casca de melancia desidratada
}

\author{
Influence of different extraction techniques on the antioxidant capacity of dehydrated watermelon \\ rind \\ Influencia de diferentes técnicas de extracción en la capacidad antioxidante de la corteza de sandía \\ deshidratada
}

Recebido: 02/10/2021 | Revisado: 10/10/2021 | Aceito: 13/10/2021 | Publicado: 15/10/2021

Marcelo Eduardo Alves Olinda de Souza

ORCID: https://orcid.org/0000-0001-6447-4131 Instituto Federal de Educação, Ciência e Tecnologia do Sertão Pernambucano, Brasil

E-mail: marcelo.olinda@ifsertao-pe.edu.br

Milena da Rocha Gomes

ORCID: https://orcid.org/0000-0001-9135-3706 Instituto Federal de Educação, Ciência e Tecnologia do Sertão Pernambucano, Brasil E-mail: miileenaagoomees@gmail.com

Nailton de Macedo Albuquerque Junior

ORCID: https://orcid.org/0000-0001-6757-5114

Universidade Federal de Campina Grande, Brasil E-mail: junior.nailton99@gmail.com

Vitória Maria Souza Candeias

ORCID: https://orcid.org/0000-0002-3092-0997 Instituto Federal de Educação, Ciência e Tecnologia do Sertão Pernambucano, Brasil E-mail: vitória.candeias@ hotmail.com

Débora Andrade Lima

ORCID: https://orcid.org/0000-0002-2338-5885 Instituto Federal de Educação, Ciência e Tecnologia do Sertão Pernambucano, Brasil E-mail: 1debora.andrade@gmail.com

Silvana Belém de Oliveira Vilar ORCID: https://orcid.org/0000-0001-8141-3373 Instituto Federal de Educação, Ciência e Tecnologia do Sertão Pernambucano, Brasil

E-mail: silvana.belem@ifsertao-pe.edu.br

Ana Beatriz Martins da Silva

ORCID: https://orcid.org/0000-0002-0495-0535 Instituto Federal de Educação, Ciência e Tecnologia do Sertão Pernambucano, Brasil E-mail: anamartins9053@gmail.com

\begin{abstract}
Resumo
A melancia é reconhecida, principalmente, pela sua elevada capacidade antioxidante natural na sua polpa e na casca, embora esta última seja descartada na sua maioria, ela possui nutrientes relevantes. O objetivo do presente trabalho foi estudar o aproveitamento da casca da melancia triturada para a obtenção de extratos com atividade antioxidante por meio do emprego de técnicas de extração como o sistema Soxhlet, maceração e extração assistida por ultrassom, utilizando solventes orgânicos de diferentes polaridades. Os extratos foram avaliados pelo rendimento do processo de extração, onde, em todas as análises, o solvente de melhor desempenho foi o etanol. Para o teor de fenólicos totais (TFT), destacou-se a técnica de extração por maceração com $3487,75 \mathrm{mg} / \mathrm{g}$, na determinação da atividade antioxidante (AA), utilizando os radicais ABTS e DPPH. O potencial antioxidante pelo método de extração por maceração foi de $45,16 \%$ e no Soxhlet foi $40,7 \%$ de inibição. Com isso, pode-se concluir que a casca de melancia é uma matéria-prima de significativo potencial antioxidante.
\end{abstract}

Palavras-chave: Aproveitamento; Nutrientes; Resíduos.

\begin{abstract}
Watermelon is mainly recognized for its high antioxidant capacity natural in its pulp and peel, although the latter is mostly discarded, it has relevant nutrients. The objective of this work was to study the use of crushed watermelon rind to obtain extracts with antioxidant activity through the use of extraction techniques such as the Soxhlet system, maceration and ultrasound-assisted extraction, using organic solvents of different polarities. The extracts were evaluated by the yield of the extraction process, where, in all analyses, the solvent with the best performance was ethanol. For the total phenolic content (TFT), the extraction technique by maceration with $3487.75 \mathrm{mg} / \mathrm{g}$ was highlighted, in the
\end{abstract}


determination of the antioxidant activity (AA), using the ABTS and DPPH radicals. The antioxidant potential by the maceration extraction method was $45,16 \%$ and in Soxhlet it was $40,7 \%$ inhibition. Thus, it can be concluded that watermelon rind is a raw material with significant antioxidant potential.

Keywords: Exploitation; Nutrients; Residue.

\section{Resumen}

La sandía es reconocida principalmente por su alta capacidad antioxidante natural en su pulpa y corteza, aunque esta última se desecha en su mayoría, tiene nutrientes relevantes. El objetivo del presente trabajo fue estudiar el uso de la corteza de sandía triturada para obtener extractos con actividad antioxidante mediante el uso de técnicas de extracción como el sistema Soxhlet, la maceración y la extracción asistida por ultrasonidos, utilizando disolventes orgánicos de diferentes polaridades. Los extractos se evaluaron por el rendimiento del proceso de extracción, donde, en todos los análisis, el disolvente con mejor rendimiento fue el etanol. Para el contenido fenólico total (TFT), la técnica de extracción por maceración destacó con 3487,75 mg/g, en la determinación de la actividad antioxidante (AA), utilizando los radicales ABTS y DPPH. El potencial antioxidante por el método de extracción por maceración fue del 45,16\% y en Soxhlet fue del 40,7\% de inhibición. Así, se puede concluir que la corteza de la sandía es una materia prima con un importante potencial antioxidante.

Palabras clave: Explotación; Nutrientes; Residuos.

\section{Introdução}

Nos últimos anos, cada vez mais atenção tem sido dada ao uso de resíduos de frutas e vegetais para diminuir o grande volume de material descartados em aterros controlados ou lixões. Aqueles resíduos, são fontes naturais de elevada importância, pois possuem uma variedade de efeitos biológicos como: fontes nutricionais, licopeno, $\beta$-caroteno, vitaminas dos complexos B e C, fibras alimentares, carboidratos, polifenóis que apresentam capacidade antioxidante e lipídeos (Sena et al., 2017; Al-Sayed \& Ahmed, 2013; Lima et al., 2010).

As melancias pertencem à família das Cucurbitáceas, e representa cerca de $20 \%$ da produção total de olerícolas no mundo (Hasanin \& Hashem, 2020; Bomfim et al.,2013). O Brasil é o quarto maior produtor mundial de melancia do mundo, perdendo apenas para China, Turquia e Irã, que são responsáveis por cerca de $80 \%$ da produção anual mundial (Oliveira et al., 2015). A melancia (Citrullus lanatusThunb. Mansf.) da variedade Crimson Sweet Extra, é uma das frutas com um cultivo mais expressivo no Brasil e possui endocarpo de grande porte, adocicado e suculento, característica estas importante para o consumidor exigente (Menezes et al., 2019).

Paralelamente a produção mundial de melancia, acarreta também a geração de grande quantidade de resíduos agrícolas, industriais e domésticas que, muitas vezes, são descartados sem o uso consciente, já que é possível a utilização do subproduto na alimentação ou na extração de compostos com diversas atividades biológicas, bem como pode ser empregado no preparo de meios de cultivos de microrganismos em processos fermentativos (Hasanin \& Hashem, 2020; Silva et al., 2018; Ogu \& Orjiakor, 2017; Martín, 2009; Marchetto et al., 2008).

Como exemplo no desenvolvimento tecnológico da cadeia produtiva alimentar, temos a produção de farinhas para incorporação parcial ou total em alimentos como, pães, bolos, cookies, biscoitos/bolachas, mingaus, sopas (Farias Silva et al., 2016; Bender et al., 2016; Piovesana et al., 2013), iogurtes, na texturização (Curti et al., 2017) e como aditivo de fermentação em bebidas (Graf et al., 2015), além de in natura como condimentos alimentícios (Menezes e Souza, 2018). A partir da elaboração de farinhas de subprodutos, estes podem ser utilizados para obtenção de extratos e/ou óleos essenciais de várias matérias-primas, onde são comumente aplicados métodos de extração como o sistema Soxhlet, maceração e extração assistida por ultrassom. Nas extrações com solventes orgânicos, são usados vários tipos de dissolvedor com diferentes polaridades como, por exemplo, o etanol, hexano e acetato de etila. Estas técnicas são aplicadas nas indústrias química, farmacêutica e de alimentos para a produção de extratos (Be-Jen et al., 2004; Pourmortazavi e Hajimirsadegui, 2007).

Sendo assim o presente trabalho avaliou a composição fenólica e o potencial antioxidante do óleo/extrato obtido da casca da melancia triturada (pó/farinha), produzidas no Submédio do Vale do São Francisco, usando diferentes técnicas de 
extração a partir de solventes distintos com a finalidade de obter um produto de maior valor agregado, com quantidades significativas de compostos bioativos.

\section{Metodologia}

O presente trabalho foi realizado no Instituto Federal de Educação, Ciência e Tecnologia do Sertão Pernambucano (IF Sertão-PE), Campus Petrolina-PE, no Laboratório Experimental de Alimentos (LEA) e Laboratório de Águas e Bebidas, seguindo a abordagem metodológica de forma quantitativa descrita por Pereira et al. (2018).

\subsection{Preparo da matéria-prima}

As melancias (Citrullus lanatusThunb. Mansf.) foram adquiridas no Submédio Vale do São Francisco em períodos de produção diferentes na região, e em seguida as frutas foram levadas para o LEA e lá foi realizado o preparo da matéria-prima: Lavando e selecionando os pomos que apresentavam cascas isentas de danos físicos. para a separação total da polpa, restando somente as cascas verdes e estas foram sanitizadas a $100 \mathrm{ppm} / 5 \mathrm{~min}$.

\subsection{Secagem e trituração da matéria-prima}

As cascas da melancia foram submetidas à desidratação no equipamento secador por circulação de ar forçado (Desidratador Pardal PE 30, 220 V, $900 \mathrm{~W}$ ) na temperatura de $60{ }^{\circ} \mathrm{C}$, sendo estes pesados a cada 60 minutos, até obtenção de peso constante.

Depois da secagem, as cascas foram trituradas em liquidificador industrial (JL COLOMBO - 8 Litros, Aço Inox) durante 05 minutos, até a obtenção do pó/farinha, que foi separada em peneira granulométrica de aço inoxidável (A Bronzinox) de 10 mesh. Logo após, o pó foi pesado, dividido em três porções com pesos distintos, embaladas em papel alumínio com saquinhos plásticos de baixa densidade e, por fim, o material foi selado e levado para a armazenagem em freezer $\left(-18^{\circ} \mathrm{C}\right)$, para a posterior etapa do processo de extração.

\subsection{Regentes}

Para a realização das extrações, foram utilizados os solventes: etanol P.A. (EtOH), que é obtido através de fontes renováveis e sendo comumente utilizado na indústria alimentícia, pois, não é toxico; hexano P.A. (Hx) que é considerado o solvente orgânico mais usado no processo de extração de óleos, por possuir estreita faixa de ebulição, ser imiscível com a água e apresentar polaridade nula.

Na Tabela 1, são apresentados os dados relacionados ao ponto de ebulição e polaridade dos solventes utilizados nas extrações.

Tabela 1. Ponto de ebulição e polaridade dos solventes utilizados no processo de extração

\begin{tabular}{ccc}
\hline Solventes & Ponto de Ebulição $^{\mathbf{1}}$ & Polaridade $^{\mathbf{2}}$ \\
\hline Etanol & $78,37^{\circ} \mathrm{C}$ & 5,2 \\
\hline Hexano & $69{ }^{\circ} \mathrm{C}$ & 0 \\
\hline
\end{tabular}

Fonte: ${ }^{1}$ Carvalho (2016); ${ }^{2}$ Souza (2015). 


\subsection{Processo de Extração}

\subsubsection{Método sistema Soxhlet}

As extraç̃oes para obtenção de extrato por sistema Soxhlet foram executadas de acordo com a metodologia do Instituto Adolfo Lutz (IAL - $4^{a}$ edição, 2008). Foram pesadas 5 gramas da casca de melancia, colocadas em cartuchos de filtro de papel (tipo: coador de café) e encaixados no equipamento 'Extrator de Óleo e Graxas' (MARCONI, MA 491). No copo de extrator, foram depositados $150 \mathrm{~mL}$ de solvente, em seguida, acoplado no sistema, tendo o aquecimento ligado de acordo com o ponto de ebulição do solvente, de forma intermitente a extração durou 6 horaso.

\subsubsection{Método Maceração}

O método da maceração consiste em uma extração da matéria-prima a temperatura ambiente em recipiente fechado, com duração de 05 dias, sob agitação manual 01 vez ao dia, utilizando solvente orgânico (Sachindra et al.,2006). Seguindo este método, utilizando frasco cor âmbar, foram adicionadas 25 gramas da amostra (casca triturada) e $100 \mathrm{~mL}$ de solvente para efetuar a extração. Passado o período estabelecido, foi realizada a filtração a vácuo com papel filtro, para remoção da amostra sólida e solvente.

\subsubsection{Método Ultrassom}

Foi utilizado o método adaptado por Gu et al. (2008), que consiste na extração da matéria-prima, a temperatura ambiente, adicionado $05 \mathrm{~g}$ de matéria-prima com $150 \mathrm{~mL}$ de solvente orgânico. Para isso, no balão volumétrico, foi depositado a amostra e o solvente, onde este recipiente foi tampado e mergulhado em uma lavadora ultrassônica, durante o período de $60 \mathrm{~min}$ de extração. Ao final, foi feita uma filtração a vácuo para separação da amostra do extrato.

\subsubsection{Eliminação do solvente por rotaevaporação}

Ao final de cada extração, a solução foi submetida ao processo de remoção do solvente, através do rotaevaporador (Fisatom 801 - Mod. 550 / 200 RPM; 230 V; 60 Hz; 1200 W, Brasil) com rotação de 60 rpm, em condições a vácuo e com temperatura próxima ou igual ao ponto de ebulição do solvente empregado. $\mathrm{O}$ extrato foi depositado em um balão de fundo chato, sob rotação, imerso no banho de água aquecida.

A rotação do frasco cria uma superfície de troca, devido ocorrer uma maior mistura, assim permitindo realizar a evaporação de maneira rápida (Souza, 2015). Após eliminação total do solvente, o extrato na sua forma pastosa, foi pesado no frasco âmbar, este último já numerado e pesado vazio antes do uso, para realização do cálculo de rendimento. O acondicionamento destes recipientes contendo as amostras foi feito em freezer a $-18^{\circ} \mathrm{C}$.

\subsection{Determinação do rendimento global das extrações $\left(\mathrm{X}_{0}\right)$}

Silva (2004) define rendimento global de extrações como a quantidade total de composto oleico extraído de um elemento, sendo obtido através de solventes, em conjunto com as condições pré-estabelecidas. Dentre estas, pode-se citar o tempo de extração e a quantidade de matéria-prima utilizada.

Foi calculado o rendimento das extrações $\left(\mathrm{X}_{\mathrm{o}}\right)$ por meio de uma equação que expressa à razão entre a massa de extrato $\left(\mathrm{m}_{\text {ext }}\right)$ obtido e a massa da farinha/pó das cascas da melancia $\left(\mathrm{m}_{\mathrm{far}}\right)$, demonstrada na Equação 1.

Equação 1. Equação para cálculo de rendimento das extrações.

$$
\text { Xo }(\%)=\frac{m_{\text {ext }}}{m_{\text {far }}} \times 100
$$


Onde:

$\mathrm{X}_{0}=$ rendimento global $(\%)$.

$\mathrm{m}_{\mathrm{ext}}=$ massa do extrato.

$\mathrm{m}_{\mathrm{far}}=$ massa da farinha/pó das cascas

\subsection{Caracterização dos compostos bioativos totais}

\subsubsection{Determinação do teor de fenólicos totais (TFT)}

Para a determinação do potencial fenólico foi utilizado o método espectrofotométrico com reagente Folin-Ciocalteu (Singleton e Rossi, 1965). As amostras reagiram em solução de carbonato de sódio 7,5\% e Folin-Ciocalteu 10\%, adaptado e validado por Natividade et al. (2013) sendo o fator de diluição igual a 10. Para os grupos hidroxi-fenólicos reduzirem o ácido fosfomolíbdico-fosfotúngstico, produzindo um complexo de coloração azul que absorve entre 620 e $740 \mathrm{~nm}$ com um comprimento de onda máximo em $725 \mathrm{~nm}$, aguardou-se $120 \mathrm{~min}$. Por fim, após a leitura no espectrofotômetro, foi utilizada a equação da regressão calculada na curva de calibração com ácido gálico, considerando as diluições, e os resultados expressos em equivalentes ao ácido gálico conforme a Equação 2.

Equação 2. Curva de calibração com ácido gálico

$$
\gamma=1019,4 X-4,6166(\mathrm{mg} / \mathrm{g})
$$

Onde:

$\mathrm{X}=$ Absorbância

\subsubsection{Determinação da capacidade antioxidante}

Por conta da sua facilidade de execução e baixo custo, além de obtenção de resultados confiáveis, o potencial antioxidante obtido pelo método do DPPH (EAG e Trolox), segundo o método de Brand-Williams et al. (1995) é bastante usado. Assim, foram adicionados $100 \mu \mathrm{L}$ da amostra a 2,9 mL de solução DPPH, aguardou-se 30 min no escuro até a leitura. Em seguida, foi obtida a equação de regressão de curva padrão elaborada com ácido gálico e trolox, conforme as Equações 3 e 4 , respectivamente.

Equação 3. Regressão ácido gálico

$$
\text { \%inibição }=\left[1-\frac{(\text { Abs Amostra })}{(\text { Abs Controle })}\right] \times 100
$$

Equação 4. Trolox (nMTrolx/l)

$$
\frac{(\% \text { inibição } o-2,5215)}{58,847} \times F D
$$

Onde:

FD = Fator de diluição

A atividade antioxidante também foi determinada pelo método de ABTS (Trolox) (2,2'-azino-bis 3-etilbenzenotiazolina-6-ácido sulfônico). O procedimento foi baseado em método desenvolvido por Miller et al. (1993) com adaptações 
feitas por Rufino et al. (2010). Sendo feito o cálculo utilizando a equação de regressão obtida de curva padrão elaborada com trolox.

\subsection{Análise Estatística}

Os resultados de rendimentos de extração, teor de fenólicos totais e da atividade antioxidantes obtidos nas amostras de casca de melancia, foram avaliados separadamente (conforme o tipo de extração e solvente utilizado) através da análise de variância (ANOVA) ao nível de 5\% de significância ( $\mathrm{p}<0,05)$, com o auxílio do software SPSS Statistics 17.0, aplicando o teste de Tukey que avalia as diferenças entre os pares de tratamentos entre si (Montgomery, 2005).

\section{Resultados e Discussões}

\subsection{Curva de secagem da amêndoa}

Ao decorrer da secagem, as cascas da melancia demonstraram uma redução gradativa de peso, a qual foi representada através de uma curva gráfica, exibida na Figura 1.

Figura 1. Curva de secagem da casca da melancia $\left(60^{\circ} \mathrm{C}\right)$.

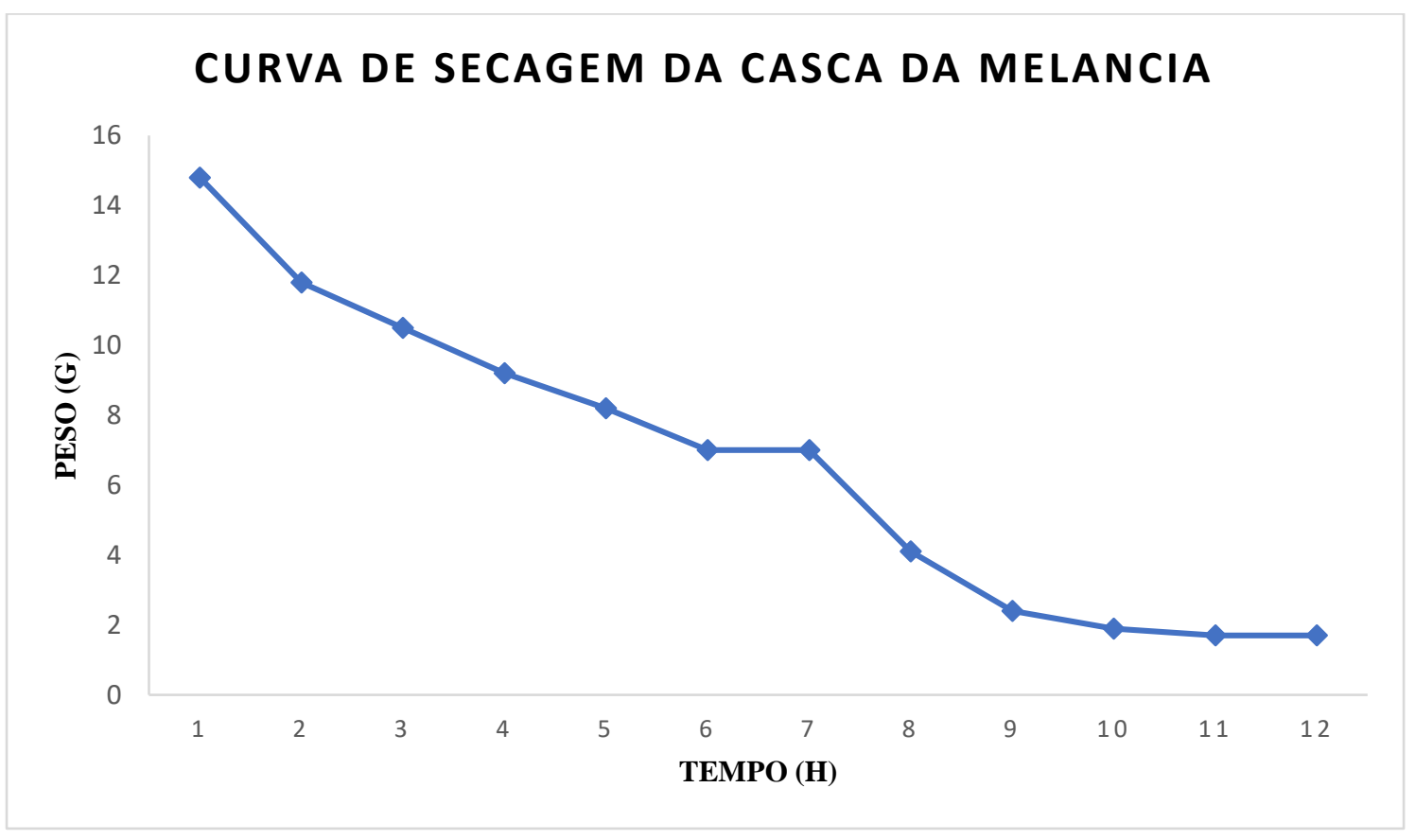

Fonte: Autores (2021).

Conforme o aumento da temperatura e o tempo de exposição, houve um aumento de pressão do vapor d'água das cascas, e a velocidade com que isso ocorre é reduzido com o passar do tempo, devido a quantidade do teor de umidade do produto ir diminuindo gradativamente. A diferença da pressão desse vapor entre o ar quente do espaço e a superfície da matéria-prima, foi gerada uma transferência de massa das cascas para o ar, ou seja, o vapor foi arrastado do produto. Assim pode-se observar a redução do teor de umidade da matéria-prima, até atingir um peso constante ocasionado pela evaporação da água, diminuição no peso e volume do material, obtendo assim a umidade de equilíbrio.

Souza et al. (2021a) ao avaliar a secagem do pó da casca de abacaxi, assim como Souza et al. (2021b) no pó da amêndoa do caroço da manga pode-se observar comportamento semelhante ao do presente estudo os quais apresentaram um tempo entre 12 e 13 horas para que toda a água presente no produto fosse transferida para o meio ocasionado pelo processo de secagem 
convectiva que ocasionou na obtenção da umidade de equilíbrio dos pós, favorecendo assim um maior tempo de conservação e armazenamento evitando que haja reações de degradação e crescimento microbiano.

\subsection{Rendimento dos Extratos}

Conforme os resultados de rendimento dos extratos (Tabela 2), foi observado que, entre os dois solventes utilizados, o hexano apresentou melhor desempenho de extração pelo sistema Soxhlet. Por outro lado, o etanol foi superior nas outras duas técnicas empregadas, conforme indicado na tabela abaixo.

Dentre os métodos utilizados, o Soxhlet mostrou o maior rendimento de extração de óleo em relação aos demais. Isso aconteceu por que a extração é realizado com aquecimento e, além disso, o solvente fica em constante circulação sob o sistema.

Tabela 2. Rendimentos dos extratos da casca de melancia desidratada.

\begin{tabular}{cccc}
\hline \multirow{2}{*}{ Solventes } & \multicolumn{3}{c}{ Metodos } \\
\cline { 2 - 4 } & Soxhlet & Ultrassom & Maceração \\
\hline Etanol & $22,75 \%$ & $8,50 \%$ & $3,61 \%$ \\
\hline Hexano & $24,18 \%$ & $3,31 \%$ & $0,61 \%$ \\
\hline
\end{tabular}

Fonte: Autores (2021).

Autores como Liu et al., (2000) sugerem que solventes com alta polaridade, como a água, e com polaridade muito baixas, ou apolares, como o hexano, não são bons extratores, porém, foi observado que a utilização do método Soxhlet combinado ao hexano, expressou um maior percentual de extrato em relação ao solvente etanol. Isso ocorreu possivelmente por que o produto obtido na extração tem mais afinidade por solvente apolar e, segundo Mattos (2013), os compostos vegetais apresentam natureza distintas e polaridades diferentes, assim para uma melhor obtenção de compostos fenólicos e atividade antioxidante, faz-se necessário uma escolha apropriada do solvente. Além disso, umas das possíveis razões é o fato do sistema de Soxhlet ser executado sob aquecimento com a temperatura de ebulição do solvente utilizado, o que fez com que a tensão superficial e viscosidade deste solvente fossem diminuídas, favorecendo a dissolução dos componentes presentes na casca da melancia.

Em relação as extrações por maceração e assistida por ultrassom, quando comparadas a do Soxhlet, foram obtidos menores rendimentos, provavelmente por serem métodos realizados em temperatura ambiente, o que fez com que a tensão superficial e viscosidade dos solventes permanecessem altas, dificultando um maior contato com os compostos solúveis e, consequentemente, reduzindo o percentual de óleo extraído.

\subsection{Compostos Fenólicos Totais}

Os resultados de teor de fenólicos totais (método de Folin-Ciocalteu) obtidos no extrato da casca de melancia desidratada, estão representados na Tabela 3.

Tabela 3. Teor de fenólicos totais do extrato da casca de melancia desidratada.

\begin{tabular}{cccc}
\hline \multirow{2}{*}{ Solventes } & \multicolumn{3}{c}{ Metodos } \\
\cline { 2 - 4 } & Soxhlet & Ultrassom & Maceração \\
\hline Etanol & $2468,35 \pm 621,44^{\mathbf{a}}$ & $2036,35 \pm 402,80^{\mathbf{a}}$ & $3487,75 \pm 445,08^{\mathbf{a}}$ \\
\hline Hexano & $529,09 \pm 99,40^{\mathbf{b}}$ & $1026,9 \pm 107,03^{\mathbf{b}}$ & $646,32 \pm 17,90^{\mathbf{b}}$ \\
\hline
\end{tabular}

Nota: Médias seguidas da mesma letra na linha, não diferem entre si pelo teste de Tukey ao nível de 5\% de probabilidade. Fonte: Autores (2021). 
Com base nos resultados expressos na tabela acima, foi constatado que dentre as metodologias usadas, a que obteve melhor média foi a extração por maceração com o etanol, tendo estatisticamente diferença significativa $(\mathrm{p}<0,05)$. Além disso, o seu coeficiente de variação foi de 12,76\% que segundo Pimentel-Gomes (1985), na ausência de referências, valores entre $10 \%$ e $20 \%$ indicam uma confiabilidade da média muito boa.

Ao analisar cascas liofilizadas das cultivares 'Manchester' e 'Smile', Costa (2017) encontrou valores de 3,97 mg/100 $\mathrm{g}^{-1}$ e $0,37 \mathrm{mg} / 100 \mathrm{~g}^{-1}$ respectivamente, tais resultados são inferiores aos valores encontrados neste estudo. Isso aconteceu, possivelmente, pelo fato de que a utilização de metodologias diferentes, além das variedades e região de cultivo que podem influenciar nos resultados de cada estudo.

O extrato da farinha/pó da casca da melancia pelo método de maceração aliada ao etanol, também apresentou valores superiores as farinhas obtidas na casca de manga (2.489 mg/ $100 \mathrm{~g}$ ) (Araújo et al., 2009), acerola (279 mg/ $100 \mathrm{~g}$ ), graviola $(24,11 \mathrm{mg} / 100 \mathrm{~g}$ ), abacaxi $(9,11 \mathrm{mg} / 100 \mathrm{~g})$ (Souza \& Vieira, 2011) e de casca de uvas (extrato acetona $75 \%$ ) que foram de 1.026,69 a 1.242,78 mg GAE/100g de peso seco nas cultivares 'Isabel' e 'Niágara', respectivamente (Soares et al., 2008). Por outro lado, o extrato obtido da farinha da casca da manga por Arbos et al., (2013) apresentou teor de compostos fenólicos significativamente superior $(6.644,68 \pm 28,17 \mathrm{mg}$ de catequina/ $100 \mathrm{~g})$.

\subsection{Capacidade Antioxidante}

A capacidade antioxidante pelos métodos de radicais livres ABTS e DPPH, obtidos no extrato da casca de melancia desidratada (Citrullus lanatusThunb. Mansf.) estão representados na Tabela 4 e 5, respectivamente.

Tabela 4. Potencial de inibição de radicais livres pelo método DPPH (mMTrolx/g).

\begin{tabular}{ccccc}
\hline \multirow{2}{*}{ Métodos } & \multicolumn{3}{c}{ Solventes } \\
\cline { 2 - 5 } & Inibição $\%$ & mMTrolx/L & Inibição $\%$ & mMTrolx/L \\
\cline { 2 - 5 } & $40,7^{\mathbf{a}}$ & $6,4995 \pm 0,68$ & $22,8^{\mathbf{b}}$ & $1,7221 \pm 0,52$ \\
\hline Soxhlet & $21,4^{\mathbf{a}}$ & $3,2088 \pm 0,27$ & $22,1^{\mathbf{a}}$ & $1,6667 \pm 0,36$ \\
\hline Ultrassom & $31,6^{\mathbf{a}}$ & $4,9488 \pm 0,39$ & $8,6^{\mathbf{b}}$ & $0,5215 \pm 0,75$ \\
\hline Maceração & & & & \multicolumn{3}{c}{ Hexano } \\
\hline
\end{tabular}

Nota: Médias seguidas da mesma letra na linha, não diferem entre si pelo teste de Tukey ao nível de 5\% de probabilidade. Fonte: Autores (2021).

A extração com o solvente etanol, por meio do sistema Soxhlet, teve maior aplicabilidade, isso porque o fator de inibição, ou seja, a atividade antioxidante, foi percentualmente maior. No trabalho de Costa (2017), ele encontrou 31,78\% e 20,53\% de capacidade antioxidante para as variedades da casca da melancia liofilizada das variedades 'Manchester' e 'Smile' respectivamente e, embora os valores sejam próximos, o resultado encontrado pelo método utilizando Soxhlet e etanol ainda foi superior.

Ao realizar um estudo com as cascas de melancias de diversas cultivares, Tarazona-Diaz et al., (2010) descobriu que a capacidade antioxidante pelo método DPPH da casca da fruta foi similar a encontrada na polpa (46.96 vs $43.46 \mathrm{mg}$ AAE $\mathrm{kg}^{-1}$ f.w). Já Tlili et al., (2011) afirma que a melancia contém antioxidantes com diversos benefícios relatados para a saúde, pois ela é rica em carotenoides como o licopeno e $\beta$-caroteno. De acordo com os estudos de Michaud et al., (2000), demonstram que o carotenoide licopeno, presente em várias frutas e hortaliças, principalmente no tomate e na melancia, é liberado somente sob altas temperaturas.

Alguns estudos têm demonstrado que existe uma forte relação positiva entre fenólicos totais e a capacidade antioxidante de frutas e hortaliças (Kaur \& Kapoor, 2002; Abdille et al., 2005) porém, em contrapartida, outros autores não têm evidenciado 
tal correlação, pois isso pode depender do método escolhido e das características hidrofóbicas ou hidrofílicas do sistema teste e dos antioxidantes testados. (Kahkonen et al., 1999; Ismail et al., 2004).

Conforme a Tabela 5, dentre os métodos usados, o que apresentou maior eficiência para a extração de compostos com atividade antioxidante por meio da análise ABTS, foi a maceração aliada ao solvente orgânico etanol. Isso porque a maceração realizada foi a temperatura ambiente (frio), ou seja, por não utilizar fontes de calor, pode ter influenciado nos resultados e eles estão de acordo com os valores de outros autores que sugerem solventes com polaridade muito baixas, ou apolares, como hexano ou diclorometano, não são bons extratores (Liu et al., 2000), mostrando assim que a amostra tem características moderadamente polares. Para a análise de atividade antioxidante na amostra submetida a extração por ultrassom com hexano, não foi possível determiná-lo, pois a quantidade de material extraído não foi suficiente para ser realizado.

Tabela 5. Potencial de inibição de radicais livres pelo método ABTS (mMTrolx/g).

\begin{tabular}{ccccc}
\hline \multirow{2}{*}{ Métodos } & \multicolumn{3}{c}{ Solventes } \\
\cline { 2 - 5 } & Inibição $\%$ & Etanol & Inibição $\%$ & mMTrolx/L \\
\cline { 2 - 5 } Soxhlet & $37,36^{\mathbf{a}}$ & $5,9232 \pm 0,82$ & $32,7^{\mathbf{b}}$ & $2,5680 \pm 0,19$ \\
\hline Ultrassom & $24,43^{\mathbf{a}}$ & $3,7270 \pm 0,28$ & N.R. & N.R. \\
\hline Maceração & $45,16^{\mathbf{a}}$ & $7,2427 \pm 0,27$ & $16,93^{\mathbf{b}}$ & $1,2226 \pm 0,06$ \\
\hline
\end{tabular}

Nota: Médias seguidas da mesma letra na linha, não diferem entre si pelo teste de Tukey ao nível de $5 \%$ de probabilidade. N.R. = Não Realizado.

Fonte: Autores (2021).

Por outro lado, segundo a capacidade de sequestrar o radical ABTS (2,2'-azinobis-3-etilbenzotiazolina-6-ácido sulfônico), Leong e Shui (2002) classificou a melancia com baixa capacidade de sequestrar, diferente da goiaba com elevada capacidade, e a laranja, mamão, manga e abacaxi com média capacidade. Estes autores ressaltaram que a contribuição do ácido ascórbico no sequestro desse radical variou extensivamente entre as frutas. No abacaxi, goiaba, mamão, melancia e laranja, a contribuição do ácido ascórbico foi de 63, 48,3, 48, 31,7 e 25\%, respectivamente, embora Wang et al. (1996) tenham relatado que o percentual de contribuição do ácido ascórbico na ação antioxidante das frutas é inferior a $15 \%$.

\section{Conclusão}

Diante dos resultados obtidos, foi observado entre as metodologias aplicadas neste trabalho para a extração dos compostos do pó/farinha da casca de melancia (Citrullus lanatusThunb. Mansf.), o método de extração por maceração utilizando o etanol como solvente, mostrou-se ter maior eficiência para a obtenção de constituintes antioxidantes e compostos fenólicos, através das análises feita pelo sequestro de radical livre ABTS e pelo Teor de Fenólicos Totais, respectivamente.

O método usando ABTS se destacou quando comparado ao DPPH por apresentar maior capacidade de sequestro do radical. Com isso, embora na literatura haja um vasto uso de diversos métodos, os valores do presente estudo mostraram-se bastante expressivos, indicando alto potencial fenólico e baixa/moderada capacidade antioxidante de compostos presentes na casca de melancia.

Dessa forma, pode-se considerar que a casca da melancia é uma matéria-prima com potencial para a obtenção de extratos com boa capacidade bioativa, com compostos que podem ser utilizados como aditivo funcional na indústria de alimentos.

Recomenda-se a utilização de outras métodos de extração, como também outros tipos de solvente, tendo em vista que a melancia (Citrullus lanatusThunb. Mansf.), é uma variedade pouco explorada e apresenta boas propriedades para a elaboração de extratos. 


\section{Agradecimentos}

Ao Instituto Federal do Sertão Pernambucano (IF-Sertão PE), pela participação do projeto de pesquisa do Programa Institucional de Bolsas de Iniciação Científica (PIBIC) e a todos os colegas que contribuíram neste trabalho.

\section{Referencias}

Abdille, M. D. H., Singh, R. P., Jayaprakasha, G. K., \& Jena, B. S. (2005). Antioxidant activity of the extracts from Dillenia indica fruits. Food Chemistry. 90 (Issue 4), 891-896.

Al-Sayed, H. M. A., \& Ahmed, A. R. (2013). Utilization of watermelon rinds and sharlyn melon peels as a natural source of dietary fiber and antioxidants in cake. Annals of Agricultural Science, 58(1), 83-95.

Araújo C. R., Melo E. A., Lima G. V. L. A., \& Maciel M. I. S. (2009). Resíduo agroindustrial de três variedades de mangas: teor de fitoquímicos e ação antioxidante. In: IX JEPEX - Jornada de ensino, pesquisa e extensão, Recife. Anais, UFRPE. p.42.

Arbos K. A., Stevani P. C., \& Castanha R. F. (2013). Atividade antimicrobiana, antioxidante e teor de compostos fenólicos em casca e amêndoa de frutos de manga. Rev. Ceres vol.60 no. 2 Viçosa Mar./Apr.

Bender, A. B. B., Luvielmo, M. M., Loureiro, B. B., Speroni, C. S., Boligon, A. A., Silva, L. P., \& Penna, N. G. (2016). Obtenção e caracterização de farinha de casca de uva e sua utilização em snack extrusado. Brazilian Journal of Food Technology, 19, 1-9.

Bomfim, I. G. A., Cruz, D. O., Freitas, B. M., \& Aragão, F. A. S. (2013). Polinização em melancia com e sem semente (1a ed., 53 p., Documentos, 168). Brasília: Embrapa Agroindústria Tropical.

Costa, Adriana Barbosa. (2017). Compostos fenólicos, capacidade antioxidante e minerais em cascas de melancias 'manchester' e 'smile' provenientes de resíduos de processamento. 45 f., il. Tese (Doutorado em Nutrição Humana)—Universidade de Brasília, Brasília.

Curti, C. A., Vidal, P. M., Curti, R. N., \& Ramón, A. N. (2017). Chemical characterization, texture and consumer acceptability of yorgurts supplemented with quinoa flour. Food Science and Technology, 37(4), 627-631.

Farias Silva, C. E., Gama, B. M. V., Oliveira, L. M. T., Araújo, L. T.; Araújo, M. L.; Oliveira Júnior, A. M.; \& Abud, A. K. S. (2016). Uso da laranja lima e seus resíduos no desenvolvimento de novos produtos. Brazilian Journal of Biosystems Engineering, 10(1), 69-96.

Graf, B. L., Rojas-Silva, P., Rojo, L. E., Delatorre-Herrera, J., Baldeón, M. E., \& Raskin, I. (2015). Innovations in health value and functional food development of Quinoa (Chenopodium quinoa Willd.). Comprehensive Reviews in Food Science and Food Safety. 14(4), 431-445.

Gu, Z., Chen, D., Han, Y., Chen, Z., \& Gu, F. (2008). Optimization of carotenoids extraction from Rhodobacter sphaeroides. LWT. 41, $1082-1088$.

Guimarães, R. R. et al. (2010). Bolos simples elaborados com farinha da entrecasca de melancia (Citrullus vulgaris, sobral): avaliação química, física e sensorial. Ciência e Tecnologia de Alimentos, 354-363.

Hasanin, M. S., \& Hashem, A. H. (2020). Eco-friendly, economic fungal universal medium from watermelon peel waste.Journal of Microbiological Methods, $168,105802$.

Instituto Adolfo Lutz (São Paulo). (2008). Métodos físico-químicos para análise de alimentos / coordenadores Odair Zenebon, Neus Sadocco Pascuet e Paulo Tiglea - São Paulo: Instituto Adolfo Lutz, p.1020, versão eletrônica.

Kaur, C., Kapoor, H. C. (2002). Anti-oxidant activity and total phenolic content of some Asian vegetables. Food Science technology. v. 37, Issue 2, February, p. $153-161$.

Khkonen, A, I., Marja, P., Hopia, Heikki, J., Vuorela, J. R., Kalevi, P. (1999). Antioxidant Activity of Plant Extracts Containing Phenolic Compounds. Journal Agricultural of Food Chemistry, 47, 3954-3962.

Leong, L. P., \& ShuI, G. (2002). An investigation of antioxidant capacity of fruit in Singapore markets. Food Chem., Washington, v.76, p.69-75.

Lima Neto, I da. S., Guimarães, I. P., Batista, P. F., Aroucha, E. M. M., \& Queiróz, M. A. (2010). Qualidade de frutos de diferentes variedades de melancia provenientes de Mossoró -RN. Revista Caatinga, 23(4), 14-20.

Liu, F. F., Ang, C. Y. W., \& Springer, D. (2000). Optimization of extraction conditions for active components in Hypericum perforatum using surface methodology. Journal of Agriculture and Food Chemistry, 48, 3.364-3.371.

Marchetto, A. M. P., Ataide, H. H., Masson, M. L. F., Pelizer, L. H., Pereira, C. H. C., \& Sendão, M. C. (2008). Avaliação das partes desperdiçadas de alimentos no setor de hortifrúti visando seu reaproveitamento. Revista Simbio-Logias, 1(2), 1-14.

Martín, P. C. (2009). El uso de residuales agroindustriales en la alimentación animal em Cuba: passada, presente y futuro. Avances en Investigación Agropecuaria, 13(3), 3-10.

Mattos, G. (2013). Extração e quantificação de ácidos fenólicos e flavonóides de eugenia pyriformiscambess usando diferentes solventes. Trabalho de conclusão de curso. Campo Mourão.

Menezes, A. C. P., \& Souza, C. F. C. (2018). Aproveitamento dos resíduos de melancia na produção de condimento para uso em alimentos. Revista Informe Goiano, 03, 1-4. 
Michaud, D. S., Feskanich, D., Rimm, E. B., Colditz, G. A., Speizer, F. E., Willett, W. C., \& Giovannucci, E. (2000). Intake of specific carotenoids and risk of lung cancer in 2 prospective US cohorts. American Journal of Clinical Nutrition, Boston, 72(4), 990-997.

Ogu, G. I., \& Orjiakor, P. I. (2017). Microbiological and nutritional qualities of fermentedmelon seed shells. International Journal of Life Sciences, 1(2), 1-9.

Oliveira, J. B., Grangeiro, L. C., Espinola Sobrinho, J. E., Moura, M. S. B., Carvalho, C. A. C. (2015). Rendimento e qualidade de frutos de melancia em diferentes épocas de plantio. Revista Caatinga, 28(2), 19-25.

Pimentel-Gomes, F. (1985). Curso de Estatística Experimental. (12a ed.), Livraria Nobel, 467p.

Piovesana, A., Bueno, M. M., \& Klajn, V. M. (2013). Elaboração e aceitabilidade de biscoitos enriquecidos com aveia e farinha de bagaço de uva. Brazilian Journal of Food Technology, 16, 68-72.

Rimando, A. M; \& Perkins-Veazie, P. M. (2005). Determination of citruline in watermelon rind. Journal of Chromatography A, 1078, 196-200.

Sachindra, N. M., Bhaskar, N., \& Mahendrakar, N. S. (2006). Recovery of carotenoids from shrimp waste in organic solvents. Waste Management, 26, 10921098 .

Santana, A. F., \& Oliveira, L. F. (2005). Aproveitamento da casca de melancia (Curcubitacitrullus, Shrad) na produção artesanal de doces alternativos.Revista de Alimentos e Nutrição,out/dez., 16(4), 363-368.

Santos, A. E. (2017). Extração de compostos bioativos do jambolão (Syzygium Cumini (L.) Skeels) a baixas pressões e livre de solvente orgânico. Dissertação (Mestrado em Engenharia de Alimentos), Florianópolis: UFSC, 2017.

Sena, D. N., Almeida, M. M. B., Nascimento, L. G. L., Brito, S. A., Sousa, P. H. M., Fernandes, \& M. F. L. (2017). Farinhas provenientes do processamento de frutas: bioacessibilidade de compostos antinutricionais. Arquivos Brasileiros de Alimentação, 2(3), 156-163.

Silva, A. M., Florentino, T. S., Medeiros, M. R. G., Silveira, A. C. M., \& Viera, V. B. (2018). Utilização integral de frutas e vegetais na elaboração de novas fontes alimentícias. International Journal of Nutrology, 11(S01), S24-S327.

Silva, D. C. de M. N. (2004). Determinação experimental de parâmetros de processos na extração supercrítica de óleo essencial de carquela (Baccaris trimera Less). Dissertação (Mestrado em Engenharia de Alimentos), Florianópolis: UFSC.

Soares, M., Welter L., Kuskosk, E.M., Gonzaga, L., \& Fett, R. (2008). Compostos fenólicos e atividade antioxidante da casca de uvas niágara e isabel. Rev. Bras. Frutic. 30(1), 059-064.

Souza M. S. B. \& Vieira L.M. (2011). Fenólicos totais e capacidade antioxidante in vitro de resíduos de polpas de frutas tropicais. Brazilian Journal of Food Technology, 14:202-210.

Souza, M. E. A. O. (2015). Potencial antioxidante de extratos da casca de manga (Mangifera indica L.) da variedade Tommy Atkins obtidos por métodos a baixa e a alta pressão e dimensionamento de uma coluna para extração supercrítica. Tese (Doutorado em Engenharia de Alimentos), Departamento de Engenharia Química e Engenharia de Alimentos, Universidade Federal de Santa Catarina.

Souza, M. E. A. O., Gomes, M. R., Candeias, V. M. S., Junior, N. M. A., Januário, E. T. F., Lima, D. A., \& Vilar, S. B. O. (2021a). Determinação da capacidade antioxidante do extrato do pó da casca do abacaxi aplicando diferentes técnicas de extração. Research, Society and Development, $10(10)$, e155101018574.

Souza, M. E. A. O., Candeias, V. M. S., Junior, N. M. A., Januário, E. T. F., Lima, D. A., Vilar, S. B. O., \& Amorim, T. A. (2021b). Capacidade Antioxidante do Extrato do pó da amêndoa do caroço da manga por diferentes técnicas de extração. Research, Society and Development, 10(9). e12010917760.

Tarazona, M., Silva-Pereira, C., Santos-Pinto, S., \& Aguayo, E. (2010). Influência de la temperatura y duracion de la conservacion en los compuestos funcionales de subproductos de pepino y sandia. VI Congr. Espanol de Ingenieria de Alimentos, Logro, 230-232.

TlilI, I., Hdider, C., Lenucci, M. S., Riadh, I., \& Jebari, H. (2011). Dalessandro, G.Bioactive compounds and antioxidant activities during fruit ripening of watermelon cultivars. Journal of Food Composition and Analysis, 24: 923-928. 